\title{
An online repository of Swift/XRT light curves of $\gamma$-ray bursts
}

\author{
P. A. Evans ${ }^{1}$, A. P. Beardmore ${ }^{1}$, K. L. Page ${ }^{1}$, L. G. Tyler ${ }^{1}$, J. P. Osborne ${ }^{1}$, M. R. Goad ${ }^{1}$, P. T. O’Brien ${ }^{1}$, L. Vetere ${ }^{2}$, \\ J. Racusin ${ }^{2}$, D. Morris ${ }^{2}$, D. N. Burrows ${ }^{2}$, M. Capalbi ${ }^{3}$, M. Perri ${ }^{3}$, N. Gehrels ${ }^{4}$, and P. Romano ${ }^{5,6}$ \\ 1 Department of Physics and Astronomy, University of Leicester, Leicester, LE1 7RH, UK \\ e-mail: pae9@star.le.ac.uk \\ 2 Department of Astronomy and Astrophysics, 525 Davey Lab., Pennsylvania State University, University Park, PA 16802, USA \\ ASI Science Data Center, ASDC c/o ESRIN, via G. Galilei, 00044 Frascati, Italy \\ 4 NASA/Goddard Space Flight Center, Greenbelt, MD 20771, USA \\ 5 INAF-Osservatorio Astronomico di Brera, via E. Bianchi 46, 23807 Merate (LC), Italy \\ 6 Università degli Studi di Milano, Bicocca, Piazza delle Scienze 3, 20126 Milano, Italy \\ Received 23 March 2007/ Accepted 18 April 2007
}

\section{ABSTRACT}

\begin{abstract}
Context. Swift data are revolutionising our understanding of Gamma Ray Bursts. Since bursts fade rapidly, it is desirable to create and disseminate accurate light curves rapidly.

Aims. To provide the community with an online repository of X-ray light curves obtained with Swift. The light curves should be of the quality expected of published data, but automatically created and updated so as to be self-consistent and rapidly available.

Methods. We have produced a suite of programs which automatically generates Swift/XRT light curves of GRBs. Effects of the damage to the CCD, automatic readout-mode switching and pile-up are appropriately handled, and the data are binned with variable bin durations, as necessary for a fading source.

Results. The light curve repository website ${ }^{\star}$ contains light curves, hardness ratios and deep images for every GRB which Swift's XRT has observed. When new GRBs are detected, light curves are created and updated within minutes of the data arriving at the UK Swift Science Data Centre.
\end{abstract}

Key words. gamma rays: bursts - gamma rays: observations - methods: data analysis - catalogs

\section{Introduction}

The data from the Swift satellite (Gehrels et al. 2004), and particularly its X-ray Telescope (XRT, Burrows et al. 2005), are revolutionising our understanding of Gamma Ray Bursts (GRBs, see Zhang 2007, for a recent review). The XRT typically begins observing a GRB $\sim 100 \mathrm{~s}$ after the trigger, and usually follows it for several days, and occasionally for months (e.g., Grupe et al. 2007). However, creating light curves of the XRT data is a nontrivial process with many pitfalls. The UK Swift Science Data Centre is automatically generating light curves of GRBs - an example light curve is given in Fig. 1 - and making them immediately available online. In this paper we detail how the light curves are created, and particularly, how the complications specific to these data are treated.

\subsection{Aspects of light curve generation}

In general, creation of X-ray light curves is a relatively simple, quick task using FTOOLS such as the XSELECT and LCMATH packages. Building Swift/XRT light curves of GRBs, however, has a number of complications which can make the task difficult and slower, as described below.

\subsubsection{GRBs fade}

The standard light curve tools, such as those mentioned above, produce light curves with uniform bin durations. Since GRBs

* http://www.swift.ac.uk/xrt_curves fade by many orders of magnitude, long-duration bins are needed at late times in order to detect the source. However, GRBs show rapid variability and evolution at early times, and short time bins are needed to resolve these features. A better approach to producing GRB light curves is to bin data based on the number of counts in a bin, rather than the bin duration. This is common practice for X-ray spectroscopy, however there are no FTOOLS available to do this for light curves. While this is our chosen means of binning GRB light curves, it is not the only option. For example, one could use the Bayesian blocks method (Scargle 1998) to determine the bin size.

Another complication caused by the fading nature of GRBs is that when the burst is bright, it is best to extract data for a relatively large radius around the GRB position, to maximise the number of counts measured. When the GRB has faded, using such a large region means that the measured counts would be dominated by background counts, making it harder to detect the source, thus it is necessary to reduce the source region size as the GRB fades. This is illustrated in Fig. 2.

\subsubsection{Swift data contain multiple observations and snapshots}

The Swift observing schedule is planned on a daily basis, and each day's observation of a given target has its own observation identification (ObsID) and event list. Thus if Swift follows a GRB for two weeks, it will produce up to fourteen event lists, all of which need to be used in light curve creation. At late times it 


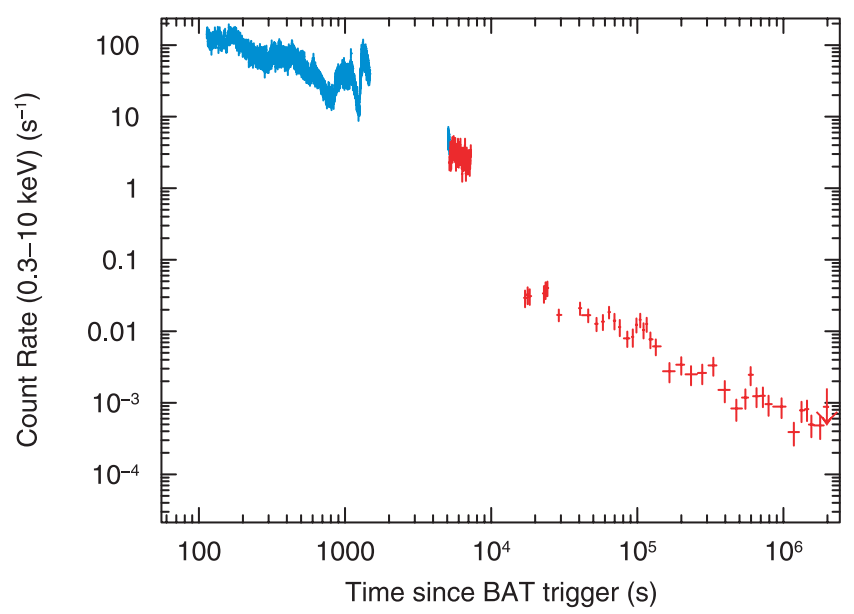

Fig. 1. Swift X-ray light curve of GRB 051117a (Goad et al. 2007), created using the software described in this paper and obtained from the Swift Light Curve Repository.

may become necessary to combine several datasets just to detect the GRB.

Also, Swift's low-Earth orbit means that it is unable to observe most targets continuously. Thus, any given ObsID may contain multiple visits to the target ("snapshots") which again will need to be combined (this differentiation between observations - datasets with a unique ObsID - and snapshots - different on-target times within an ObsID - will be used throughout this paper). Combining snapshots/observations can result in bins on a light curve where the fractional exposure is less than 1 . This must be taken into account in calculating the count rate.

The standard pipeline processing of Swift data ${ }^{1}$ ensures that the sky coordinates are correctly attained for each event, however the position of the GRB on the physical detector can be different each snapshot due to changes in the spacecraft attitude. This becomes a problem when one considers the effects of bad pixels and columns.

\subsubsection{CCD Damage}

On 2005-May-27 the XRT was struck by a micrometeoroid (Abbey et al. 2005). Several of the detector columns became flooded with charge ("hot"), and have had to be permanently screened out. Unfortunately, these lie near the centre of the CCD, so the point spread function (PSF) of a GRB often extends over these bad columns. As well as these columns there are individual "hot pixels" which are screened out, and other pixels which become hot when the CCD temperature rises, so may be screened out in one event list, but not in the next. Exposure maps and the XRTMKARF tool can be used to correct for this, however this has to be done individually for each Swift snapshot (since the source will not be at the same detector position from one snapshot to the next). A single day's observation contains up to 15 snapshots, thus to do this manually is a slow, laborious task. The forthcoming XRTLCCORR program should make this process easier, however it will still need to be executed for each observation.

\footnotetext{
${ }^{1}$ http://swift.gsfc.nasa.gov/docs/swift/analysis/ xrt_swguide_v1_2.pdf
}
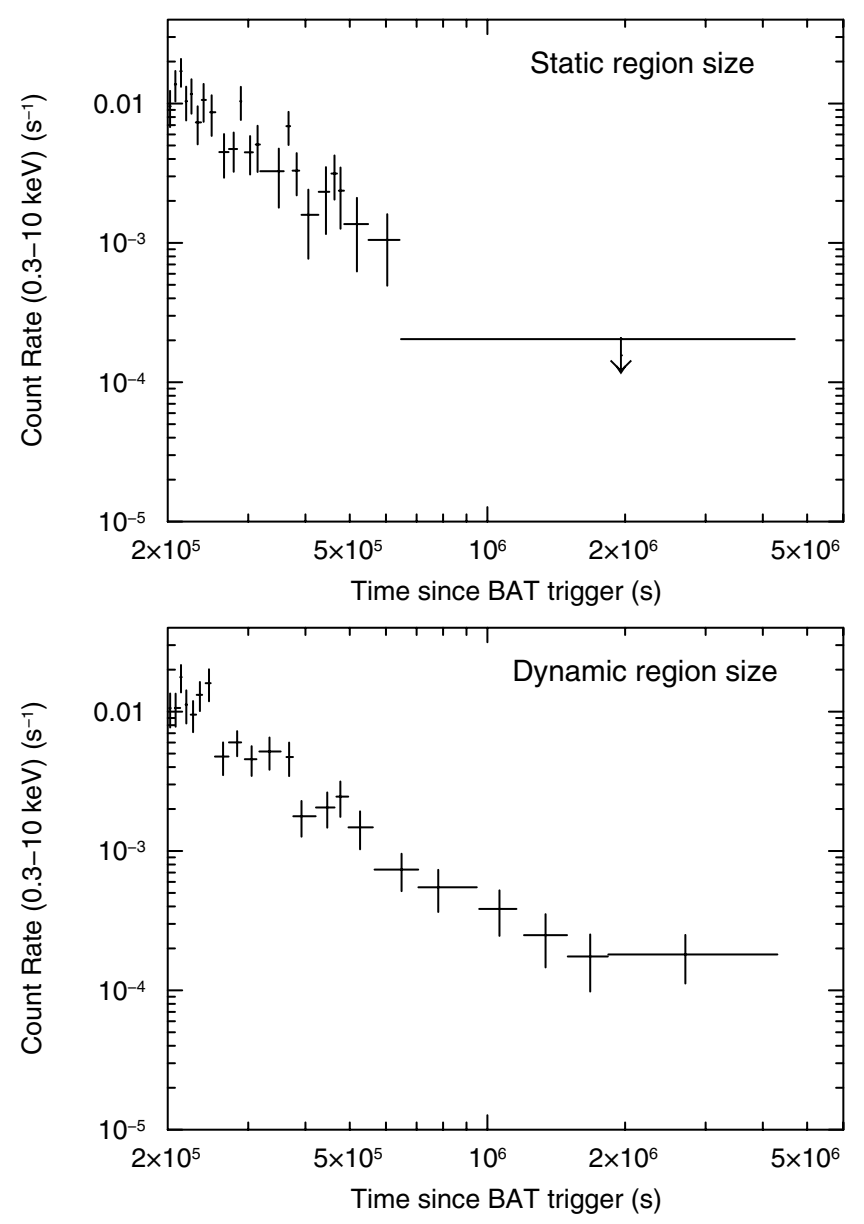

Fig. 2. Late-time Swift X-ray light curves of GRB 060614 (Mangano et al. 2007), showing the need for the source region to be reduced as the data fades. Top panel: where the source extraction region remains large at late times, the source cannot be detected after $600 \mathrm{ks}$. Bottom panel: using a smaller source extraction region at later times suppresses the background, yielding 6 more datapoints on the light curve.

\subsubsection{Automatic readout-mode switching}

One of the XRT's innovative features is that it changes readout mode automatically depending on the source intensity (Hill et al. 2004). At high count-rates it operates in Windowed Timing (WT) mode, where some spatial information is sacrificed to gain time resolution $\left(\Delta t=1.8 \times 10^{-3} \mathrm{~s}\right)$. At lower count-rates Photon Counting (PC) mode is used, yielding full spatial information, but lower time resolution $(\Delta t=2.5 \mathrm{~s})$. The XRT also has Photodiode (PD) mode, which contains no spatial information, but has very high time resolution $\left(\Delta t=1.4 \times 10^{-4} \mathrm{~s}\right)$. This mode was designed to operate for higher count-rates than WT mode, however it was disabled following the micrometeoroid impact. Prior to this, the XRT produced very few PD mode frames before switching to WT so we have limited our software to WT and PC modes.

For a simple, decaying GRB the earliest data are in WT mode and as the burst fades the XRT switches to PC mode. This is not always the case; the XRT can toggle between modes. GRB 060929 for example, had a count-rate of $\sim 0.1$ counts $\mathrm{s}^{-1}$ and the XRT was in PC mode, when a giant flare pushed the count-rate up to $\sim 100$ counts $\mathrm{s}^{-1}$ and the XRT switched into WT mode, causing a $200 \mathrm{~s}$ gap in the PC exposure (Fig. 3, upper panel). Since the initial CCD frames are taken in WT mode, and the PC data both preceded and succeeded the WT data taken 

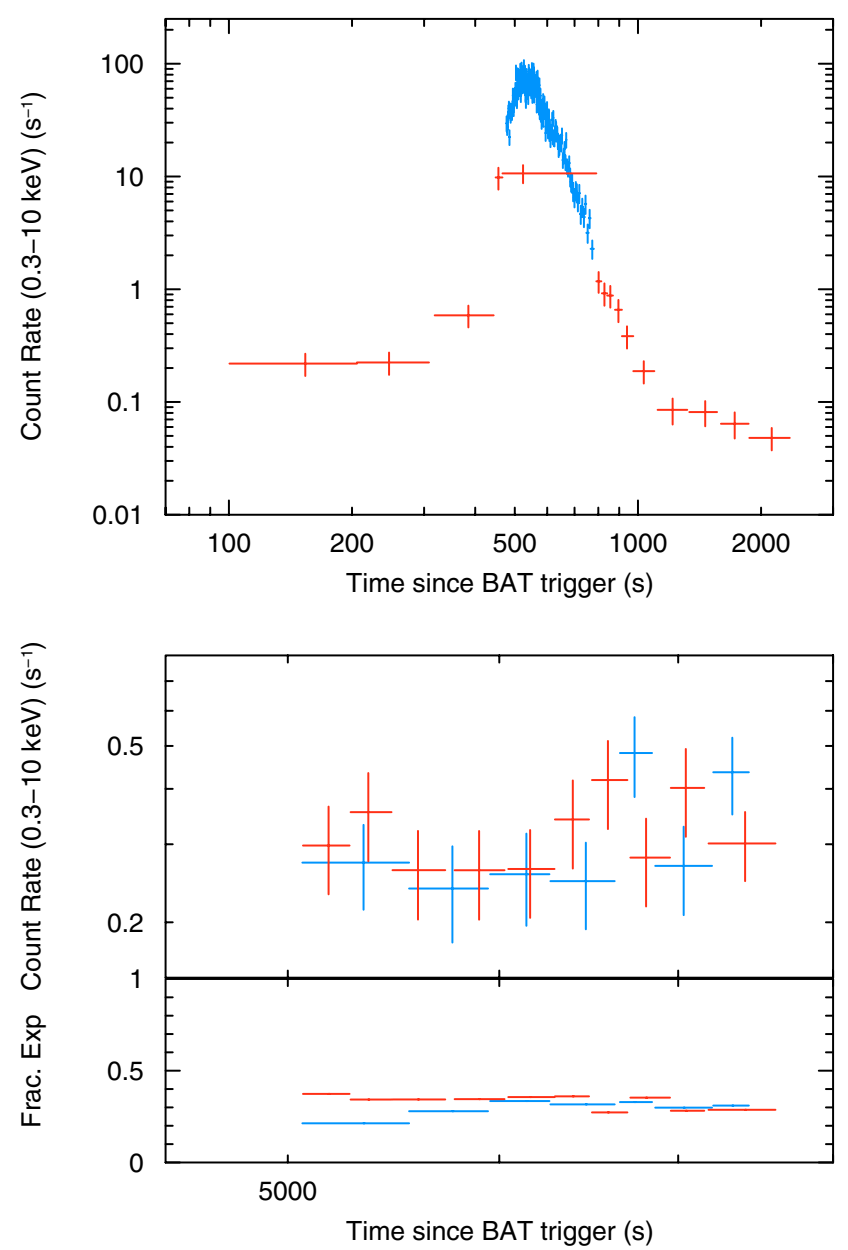

Fig. 3. Swift X-ray light curves of two GRBs, showing the switching between readout modes. WT mode is blue, PC mode red. Top panel: GRB 060929. The XRT changed from PC to WT mode due to a large flare. Bottom panel: GRB 050315. The XRT was "mode-switching" during the second snapshot. The lower pane shows the fractional exposure, which is highly variable due to this effect.

during the flare, there are large overlaps between the WT and $\mathrm{PC}$ data.

On occasions, such as when Swift was observing GRB 050315 (Vaughan et al. 2006), the XRT oscillates rapidly between WT and PC modes (Fig. 3, lower panel). This "mode switching" occurs when the count-rate in the central window of the CCD changes rapidly. Such variation is usually due to the rapid appearance and disappearance of hot pixels at high $\left(\sim-52{ }^{\circ} \mathrm{C}\right) \mathrm{CCD}$ temperatures (the XRT is only passively cooled due to the failure of the on-board thermoelectric cooler, Kennea et al. 2005), although contamination by photons from the illuminated face of the Earth can also induce mode switching. Recent changes in the on-board calibration have significantly reduced the effects of hot-pixel induced mode switching, however when it does happen it complicates light curve production by causing a variable fractional exposure. Also, during mode switching the XRT does not stay in either mode long enough to collect sufficient data to produce a light curve bin (see Sect. 2.2), thus the WT and PC bins can overlap. The lower panel of Fig. 3 illustrates these points.

\subsubsection{Pile-up}

Pile-up occurs when two photons are incident upon on the same or adjacent CCD pixels in the same CCD frame. Thus, when the detector is read out the two photons are recorded as one event. Pile-up in the Swift XRT has been discussed by Romano et al. (2006) for WT mode and Vaughan et al. (2006) and Pagani et al. (2006) for PC mode. Their quantitative analyses show the effects of pile-up at different count rates, and we used these values to determine when we consider pile-up to be a problem (see Sect. 2.2).

This problem is not unique to Swift, but because GRBs vary by many orders of magnitude, pile-up must be identified and corrected in a time-resolved manner. The standard way to correct for pile-up is to use an annular source extraction region, discarding the data near the centre of the PSF where pile-up occurs. For constant sources, or those which vary about some roughly constant mean, it is usually safe to use this annular region at all times. This is not true for GRBs, which can span five decades in brightness; using an annulus when the burst is faint would make it almost undetectable!

In the following sections we detail the algorithm used to generate light curves automatically, and in particular we concentrate on how the above issues are resolved.

\section{Light curve creation procedure}

The raw Swift/XRT data are processed at the Swift Data Center at NASA's Goddard Space Flight Center, using the standard Swift software developed at the ASI Science Data Center (ASDC) in Italy. The processed data are then sent to the Swift quick-look archives at Goddard, the ASDC, and the UK. As soon as data for a new GRB arrive at the UK site, the light curve generation software is triggered, and light curves made available within minutes.

The light curve creation procedure can be broken down into three phases. The preparation phase gathers together all of the observations of the GRB, creating summed source and background event lists. The production phase converts these data into time-binned ASCII files, applying corrections for the abovementioned problems in the process. The presentation phase then produces light curves from the ASCII files, and transfers them to the online light curve repository.

\subsection{Phase \#1 - Preparation phase}

In overview: this phase collates all of the observations, defines appropriate source and background regions (accounting for pileup where necessary), and ultimately produces a source event list and background event list for WT and PC mode, which are then passed to the production phase.

The preparation phase begins by creating a list of ObsIDs for the GRB, and then searching the file metadata to ascertain the position of the burst, the trigger time, and the name. An image is then created from the first PC-mode event list and the FTOOL XRTCENTROID is used to obtain a more accurate position. A circular source region is then defined, centred on this position, and initially 30 pixels $\left(71^{\prime \prime}\right)$ in radius. A background region is also defined, as an annulus centred on the burst with an inner radius of 60 pixels $\left(142^{\prime \prime}\right)$ and an outer radius of 110 pixels $\left(260^{\prime \prime}\right)$. The image is also searched for serendipitous sources close to the GRB (e.g. there is a flare star 40" from GRB 051117A; Goad et al. 2007), and if any are found to encroach on the 
Table 1. Source extraction radii used for given count rates. $R$ is the measured, uncorrected count rate.

\begin{tabular}{cc}
\hline \hline Count rate $R\left(\right.$ counts s $\left.^{-1}\right)$ & Source radius in pixels $(\operatorname{arcsec})$ \\
\hline$R>0.5$ & $30\left(70.8^{\prime \prime}\right)$ \\
$0.1<R \leq 0.5$ & $25\left(59.0^{\prime \prime}\right)$ \\
$0.05<R \leq 0.1$ & $20\left(47.2^{\prime \prime}\right)$ \\
$0.01<R \leq 0.05$ & $15\left(35.4^{\prime \prime}\right)$ \\
$0.005<R \leq 0.01$ & $12\left(28.3^{\prime \prime}\right)$ \\
$0.001<R \leq 0.005$ & $9\left(21.2^{\prime \prime}\right)$ \\
$0.0005<R \leq 0.001$ & $7\left(16.5^{\prime \prime}\right)$ \\
$R \leq 0.0005$ & $5\left(11.8^{\prime \prime}\right)$ \\
\hline
\end{tabular}

source region, the source extraction radius is reduced to prevent contamination.

The software then takes each event list in turn. The bad pixel information is obtained from the "BADPIX" FITS extension and stored for use in the production phase. An image of the background region is created, and the DETECT routine in XIMAGE is used to identify any sources with a count-rate $\geq 3 \sigma$ above the background level. For each source thus found, a circular region centred on the source with a radius equal to the source extent returned by XIMAGE, is excluded from the background region.

The event list is then broken up into individual snapshots, the mean count-rate during each snapshot is ascertained and used to determine an appropriate source region size (Table 1) and appended to the event list for later use. The values in Table 1 were determined by manual analysis of many GRB observations, and reflect a compromise between minimising the background level while maximising the proportion of source counts that are detected.

For each snapshot, the detector coordinates of the object are found using the POINTXFORM FTOOL, and used to confirm that both source and background regions lie within the CCD. If the background region falls off the edge of the detector it is simply shifted by an appropriate amount (ensuring that the inner ring of the annulus remains centred on the source). The source region must remain centred on the source in order for later count-rate corrections to be valid, however if this results in part of the extraction region lying outside of the exposed CCD area, the source region for this snapshot is reduced.

The first part of pile-up correction is carried out at this stage. A simple, uniform time-bin light curve is created with bins of $1 \mathrm{~s}$ $(5 \mathrm{~s})$ for WT (PC) mode, and then parsed to identify times where the count rate climbs above $150(0.6)$ counts $^{-1}$; such times are considered to be at risk of pile-up. For WT mode we are unable to investigate further, since we have only one-dimensional spatial information arranged at an arbitrary (albeit known) angle in a two dimensional plane, and no tools currently exist to extract a PSF from such data. Instead, the centre of the source region is excluded, such that the count rate in the remaining pixels never rises above 150 counts $\mathrm{s}^{-1}$. The number of excluded pixels is typically in the range $\sim 6-20$, depending on the source brightness. For PC mode, a PSF profile is obtained for the times of interest, and the wings of this (from 25" outwards) are fitted with a King function which accurately reproduces Swift's PSF (Moretti et al. 2005). This fit is then extrapolated back to the PSF core, and if the model exceeds the data by more than the 1- $\sigma$ error on the data, the source is classified as piled up (Fig. 4). The source region is then replaced with an annular region whose inner radius is that at which the model PSF and the data agree to within 1- $\sigma$ of the data. Note that these annular regions are only used during the intervals for which pile-up was detected, the rest of the time a

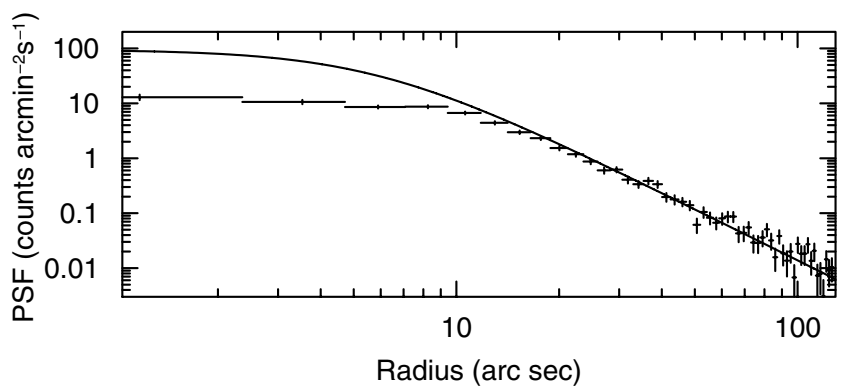

Fig. 4. The PSF of GRB 061121 (Page et al. 2007), during the first snapshot of PC data. The model PSF was fitted to the data more than $25^{\prime \prime}$ from the burst. The central $10^{\prime \prime}$ are clearly piled up.

circular region is used (or a box-shaped region for WT mode). If there are several separate intervals of pile-up (e.g., pile-up lasts for several snapshots, or a flare causes the count-rate to rise into the pile-up régime), they each have their own annular region. The inner radii of the annuli (or size of the excluded region in WT mode) are stored in the event list, so that in the production phase the count-rate can be corrected for events lost by the exclusion of the central part of the PSF.

The time-dependent region files thus created are used to generate source and background event lists for this snapshot. This process is performed for every snapshot in every observation of the GRB, and the event lists are then combined to yield one source and one background event list for each XRT mode. Additionally, all PC-mode event lists are merged for use in the presentation phase (phase \#3)

\subsection{Phase \#2 - Production phase}

In this phase the data are first filtered so that only events with energy in the range $0.3-10 \mathrm{keV}$ are included. For WT (PC) mode, only events with grades 0-2 (0-12) are accepted. Each mode is then processed separately: WT and PC mode data are not merged. The process described in this section occurs three times in parallel: once on the entire dataset, once binning only soft photons (with energies in the range $0.3-1.5 \mathrm{keV}$ ), and once binning only the hard photons $(1.5-10 \mathrm{keV})$. The data are then binned and background subtracted. Since the source region is dynamic and could change within a bin, each background photon is individually scaled to the source area (the source radius used was saved in each event list during the preparation phase).

A bin (i.e. a point on the light curve) is defined as the smallest possible collection of events which satisfies the following criteria:

- There must be at least $C$ counts from the source event list.

- The bin must span at least $0.5(2.51) \mathrm{s}$ in WT (PC) mode.

- The source must be detected at a significance of at least $3 \sigma$.

- There must be no more events within the source region in this CCD frame.

For the energy-resolved data, both the soft and hard data must meet these criteria individually to complete a bin.

$C$, the minimum number of counts in the source region, is a dynamic parameter. Its default value of 30 for WT mode and 20 for PC mode is valid when the source count-rate is one count per second. It scales with count rate, such that an order of magnitude change in count rate produces a factor of 1.5 change in $C$. This is done discretely, i.e. where $1 \leq$ rate $<10, C=30$ counts (WT mode), for $10 \leq$ rate $<100, C=45$ counts etc. $C$ must always be above 15 counts, so that Gaussian statistics remain 
valid. Note that $C$ always refers to the number of measured counts, with no corrections applied, however "rate" refers to the corrected count rate (see below). These values of $C$ give poor signal-to-noise levels in the hardness ratio, so for the energyresolved data we require $2 C$ counts in each band in order to create a bin.

The second criterion (the bin duration) is in place to enable reasonable sampling of the background. For the third criterion we define the detection significance as $\sigma=N / \sqrt{B}$, where $N$ is the number of net counts from the source and $B$ is the number of background counts scaled to the source area. Thus we require that a datapoint have a $<0.3 \%$ probability of being a background fluctuation before we regard it as "real".

The final criterion is used because the CCD is read out at discrete times, thus all events that occur between successive readouts (i.e. within the same frame) have the same time stamp. Thus, if the final event in one bin and the first event in the next were from the same frame, those bins would overlap. Apart from being cosmetically unpleasant, this will also make modelling the light curves much harder, and is thus avoided.

At the end of a Swift snapshot, there may be events left over which do not yet comprise a full bin. These will be appended to the last full bin from this snapshot, if there is one, otherwise they are carried over to the next snapshot. At the end of the event list, if there are still spare events, this bin is replaced with an upper limit on the count rate. This is calculated at the $3 \sigma$ (i.e. 99.7\%) confidence level, using the Bayesian method championed by Kraft et al. (1991).

As the data are binned and background subtracted, the countrates are corrected for losses due to pile-up, dead zones on the CCD (i.e. bad pixels and bad columns) and source photons which fell outside the source extraction region. This correction, which is applied on an event-by-event basis, is achieved by numerically simulating the PSF for the relevant XRT mode over a radius of 150 pixels, and summing it. It is then summed again, however this time, the value of any pixel in the simulated PSF which corresponds to a bad pixel in the data is set to zero before the summation (the lists of bad pixels and the times for which they were bad were saved in the preparation phase). Furthermore, only the parts of the PSF which were within the data extraction region are included. Taking the ratio of the complete PSF to the partial PSF gives the correction factor. This method is analogous to using exposure maps and the XRTMKARF task, as is done when manually creating light curves. Alternative methods of using XRTMKARF give correction factors which differ by up to 5\%; we compared our correction factors with these, and found them to lie in the middle of this distribution.

In addition to these corrections, we need to ensure that the exposure time is calculated correctly: mode switching, or bins spanning multiple snapshots, will result in a bin duration which is much longer than the exposure time. This is done by using the Good Time Interval (GTI) information from the event lists: if a bin spans multiple GTIs the dead-times between GTIs are summed, and the result is subtracted from the bin duration to give the exposure time, which is used to calculate the count rate. The fractional exposure is defined as the exposure time divided by the bin duration.

Finally, the data are written to ASCII files. The following information is saved for each bin:

- Time in seconds (with errors). The bin time is defined as the mean photon arrival time, and the (consequentially asymmetric) errors span the entire time interval covered by the bin. Time zero is defined as the BAT trigger time. For
non-Swift bursts, the trigger time given in the GCN circular which announced the GRB is used as time zero.

- Source count rate (and error) in counts $\mathrm{s}^{-1}$. This is the final count rate, background subtracted and fully corrected, with $\mathrm{a} \pm 1-\sigma$ error.

- Fractional exposure.

- Background count rate (and error) in counts $\mathrm{s}^{-1}$. This is the background count rate scaled to the source region, with a $\pm 1-\sigma$ error.

- Correction factor applied to correct for to pile-up, dead zones on the CCD, and source photons falling outside of the source extraction region.

- Measured counts in the source region.

- Measured background counts, scaled to the source region.

- Exposure time.

- Detection significance $(\sigma)$, before corrections were applied.

If an upper limit is produced, the measured counts and detection significance columns refer to the data which have been replaced with an upper limit. The significance of the upper limit is always $3 \sigma$.

$\sigma$ is always calculated before the corrections are applied, since it is a measure of how likely it is that the measured counts, not corrected counts, were caused by a fluctuation in the background level.

\subsubsection{Counts to flux conversion}

The conversion from count rates (as in our light curves) to flux requires spectral information. Since automatic spectral fitting is prone to errors (e.g. due to local minima of the fit statistic), we refrain from doing this. Furthermore, accurate flux conversion needs to take into account spectral variation as the flux evolves, which is beyond the scope of this work.

The GCN reports issued by the Swift team contain a mean conversion factor for a given burst. These tend to be around $5 \times 10^{-11} \mathrm{erg} \mathrm{cm}^{-2}$ count $^{-1}(0.3-10 \mathrm{keV})$, suggesting such a value could be used as an approximate conversion. For 10 Swift bursts between GRB 070110 and GRB 070306, the mean flux conversion is $5.04 \times 10^{-11} \mathrm{erg} \mathrm{cm}^{-2}$ count $^{-1}$, with a standard deviation of $2.61 \times 10^{-11} \mathrm{erg} \mathrm{cm}^{-2}$ count $^{-1}$.

\subsection{Phase \#3 - Presentation phase}

The final phase parses the output of the production phase to produce light curves. Three such curves are produced, and Fig. 5 shows an example of each; count-rates and the time since trigger are plotted logarithmically. The first is a basic light curve, simply showing count-rate against time. The second also shows the background level and fractional exposure. In WT mode when the GRB is bright, the background tends to be dominated by the $<1 \%$ of the PSF which leaks into the background region, but because of the high source count rate, this has negligible effects on the corrected count rates. The PC mode background should generally be approximately constant. If it shows large variations, the data may be contaminated by enhanced background linked to the sunlit Earth. Unfortunately, such contamination is currently unpredictable and varies both spatially and temporally; it is thus very difficult to correct for manually, and our automated processing does not currently correct for this. PC mode data points which occur during times of variable background should thus be treated with caution. We note, however, that our testing procedure (Sect. 3) does not show our light curves to be degraded when bright Earth characteristics become apparent. 

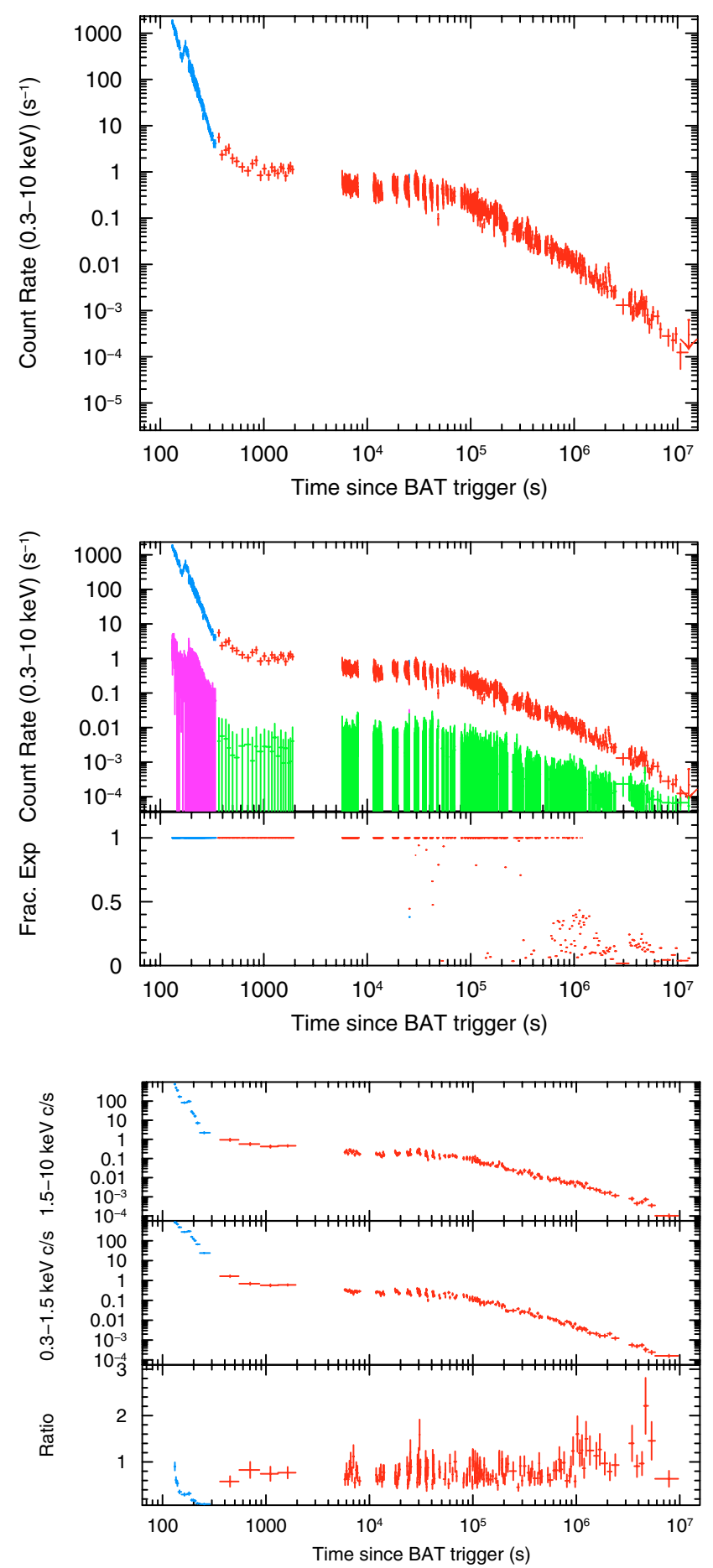

Fig. 5. Light curve images for GRB 060729. These data have been discussed by Grupe et al. (2007). Top panel: basic light curve. Centre panel: detailed light curve, with background levels shown below the light curve, and fractional exposure given in the lower pane. Bottom panel: hardness ratio. The 3 panes are (top to bottom) hard: $(1.5-10 \mathrm{keV})$, soft $(0.3-1.5 \mathrm{keV})$ and the ratio (Hard/Soft).

The third light curve produced in this phase is energyresolved. The hard- and soft-band light curves are shown separately, and the hard/soft ratio makes up the bottom panel of this plot.

Also created in this phase is a deep PC mode image, using the summed PC event list created in the preparation phase. This image is split into three energy bands: $0.3-1.2 \mathrm{keV}, 1.2-1.8 \mathrm{keV}$

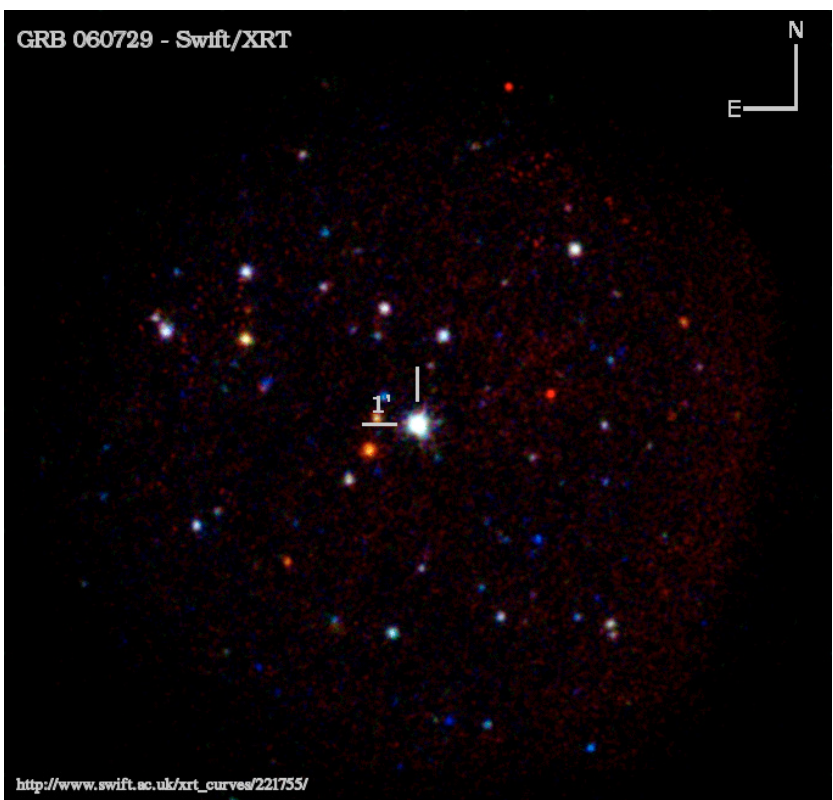

Fig. 6. An example three colour image. This is GRB 060729, and the exposure time is $1.2 \mathrm{Ms}$.

and $1.8-10 \mathrm{keV}$. These bands were chosen based on the spectra of the GRBs seen by Swift to date, to ensure that for a "typical" burst, there will be approximately equal numbers of counts in each band. These three energy-resolved images are plotted on a logarithmic scale, and combined (using IMAGEMAGICK to produce a 3-colour image (with red, green and blue being the soft, medium and hard bands respectively). This is then smoothed using IMAGEMAGICK.

Once created, these products are transferred to the online repository.

\subsection{Immediate light curve regeneration}

Our light curve generation is a dynamic process: a light curve is created when the first XRT data arrive in the UKSSDC archive - typically $1.5-2 \mathrm{~h}$ after the burst - and it is then updated whenever new data have been received and undergone the pipeline processing. Thus, a light curve should never be more than $\sim 15$ min older than the quick-look data. If the GRB is being observed every orbit, new data can be received as often as every $96 \mathrm{~min}$.

The update procedure is identical to that described in Sects. 2.1-2.3 above, except that only the new data are processed and the results appended to the existing light curve. In the case where the existing light curves ends with an upper limit, the data from this upper limit are reprocessed with the new data, hopefully enabling that limit to be replaced with a detection.

\section{Testing procedure}

In order to confirm that our light curves are correct, we used manually created light curves for every GRB detected by the Swift XRT up to GRB 070306, which had been produced by one of us (K.L. Page). We broke each light curve up into phases of constant (power-law) decay, and compared the count-rate and time at the start and end of each of these phases. We also confirmed that the shape of the decay was the same in both automatic and manually created light curves. Where applicable, we also confirmed that the transition between XRT read-out modes 
looked the same in both sets of light curves. Once we were satisfied that our light curves passed this test, we also compared a random sample of 30 GRBs with those manually created by other members of the Swift/XRT team and again found good agreement.

\section{Data availability and usage}

Our light curve repository is publicly available via the internet, at: http: //www.swift.ac.uk/xrt_curves/

Specific light curves can be accessed directly by appending their Swift target ID to this URL ${ }^{2}$.

While every effort has been made to make this process completely automatic, there may be cases where the light curve generation fails (e.g. if the source is too faint to centroid on, or if there are multiple candidates within the BAT error circle). In this event, a member of the Swift/XRT team will manually instigate the creation procedure as soon as possible. For GRBs detected by other observatories which Swift subsequently observes, the creation procedure will not be automatically triggered, however the XRT team will trigger it manually in a timely manner.

These light curves, data and images may be used by anyone. In any publication which makes use of these data, please cite this paper in the body of your publication where the light curves are presented. The suggested wording is:

"For details of how these light curves were produced, see Evans et al. (2007)".
Please also include the following paragraph in the Acknowledgements section:

"This work made use of data supplied by the UK Swift Science Data Centre at the University of Leicester."

Acknowledgements. P.A.E., A.P.B., K.L.P., L.G.T. and J.P.O. acknowledge PPARC support. L.V., J.R., P.M. and D.N.B. are supported by NASA contract NAS5-00136.

\section{References}

Abbey, A. F., Carpenter, J., Read, A., et al. 2005, in ESA-SP 604, The X-ray Universe 2005, 943

Burrows, D. N., Hill, J. E., Nousek, J. A., et al. 2005, Sp. Sci. Rev., 120, 165

Gehrels, N., Chincarini, G., Giommi, P., et al. 2004, ApJ, 611, 1005

Goad, M. R., Page, K. L., Godet, O., et al. 2007, A\&A, 468, 103

Grupe, D., Burrows, D. N., Patel, S. K., et al. 2007, ApJ, in press [arXiv: astro-ph/0611240]

Hill, J. E., Burrows, D. N., Nousek, J. A., et al. 2004, SPIE, 5165, 217 Kraft, R. P., Burrows, D. N., Nousek, J. A., et al. 1991, ApJ, 374, 344 Kennea, J. A., Burrows, D. N., Wells, A., et al. 2005, SPIE, 5898, 329 Mangano, V., Holland, S. T., Malesani, D., et al. 2007, A\&A, in press Moretti, A., Campana, S., Mineo, T., et al. 2005, SPIE, 5898, 360 Pagani, C., Morris, D. C., Kobayashi, S., et al. 2006, ApJ, 645, 1315 Page, K. L., Willingale, R., Osborne, J. P., et al. 2007, ApJ, in press [arXiv:astro-ph/0704.1609]

Romano, P., Campana, S., Chincarini, G., et al. 2006, A\&A, 456, 917 Scargle, J. D. 1998, ApJ, 504, 405

Vaughan, S., Goad, M. R., Beardmore, A. P., et al. 2006, ApJ, 638, 920

Zhang, B. 2007, ChJAA, 7, 1

${ }^{2}$ The target ID is the trigger number, given in the GCN notices and circulars, but padded with leading zeroes to be 8 digits long. e.g. GRB 060729 had the trigger number 221755 , so its target ID is 00221755 . 